\title{
Abejas y Covid-19: una regulación jurídica necesaria*
}

\author{
Marita Giménez-Candela \\ Catedrática. \\ Directora del ICALP y del Máster en Derecho Animal (UAB) \\ ORCID: 0000-0002-0755-5928 \\ SCOPUS ID: 57218923647
}

Recepción: Diciembre 2020

Aceptación: Diciembre 2020

Cita recomendada. Giménez-Candela, M., Abejas y Covid-19: una regulación jurídica necesaria, dA. Derecho Animal (Forum of Animal Law Studies) 11/4 (2020). - DOI https://doi.org/10.5565/rev/da.558

\section{Resumen}

Las abejas llevan siglos sobrevolando los textos jurídicos. De hecho, son los únicos animales a los que los Códigos continentales dedican una atención específica y cuyo régimen jurídico ha permanecido inalterado, a pesar de los muchos cambios que se han producido en el entorno social, económico y biológico de nuestro planeta. La progresiva desaparición de las abejas junto a la profunda crisis que ha provocado el coronavirus, obliga a plantearse una nueva visión de la salud personal y colectiva, en la que la protección de la biodiversidad es ineludible. Los insectos polinizadores son clave para el mantenimiento de la salud del planeta y del equilibrio de los ecosistemas. De ellos depende nuestra alimentación y el mantenimiento de la salud, en gran medida. Este trabajo, quiere sentar las bases para una nueva consideración de las abejas en el ámbito jurídico. La UE es consciente de que la legislación relativa es insuficiente y obsoleta. Más protección de las abejas, equivale a mejor protección de la salud.

Palabras clave: abejas; Coronavirus; Covid-19; insectos polinizadores; biodiversidad; bioseguridad; salud personal; salud colectiva; derecho de las abejas; miel; comercio de la miel; panales; colmenas; enjambres; productos melíferos; apicultura; etiquetaje; abejas en el CC; abejas en el derecho continental.

\section{Abstract - Bees and Covid-19: a necessary legal regulation}

Bees have been hovering under the radar of legal texts for centuries. In fact, they are the only animals to which continental codes dedicate specific attention and whose legal framework has remained unaltered, despite many changes that have come about in the social, economic and biological setting of our planet. The progressive disappearance of bees combined with the profound crisis caused by the coronavirus obliges the consideration of a new vision of personal and collective health in which biodiversity protection is inevitable. Pollinating insects are key for the health of the planet and the balance of ecosystems. To a great extent our diet and health maintenance rely on them. This work seeks to lay the basis for a new consideration of bees in the legal realm. The EU is aware that the relative legislation is insufficient and obsolete. More protection for bees equates to better health protection.

Key words: bees; Coronavirus; Covid-19; pollinating insects; biodiversity; biosecurity; personal health; collective health; the rights of bees; honey; the trading of honey; honeycombs; hives; swarms; products containing honey; apiculture; labelling; bees in the $\mathrm{CC}$; bees in continental law. 


\section{Sumario}

1. Las abejas en las Fuentes históricas

1.1. Propiedad

1.2. Comercio de la miel y productos derivados

2. Las abejas en los Códigos continentales

3. Las abejas y la biodiversidad

\section{Las abejas en las Fuentes históricas}

Una primera observación, se refiere a la abundancia de testimonios de todo tipo que, acerca de la miel y de la apicultura, encontramos en las fuentes históricas, lo que evidencia la gran relevancia que las abejas y los productos melíferos tuvieron en la Antigüedad, no sólo en la alimentación, la medicina, la cosmética o la religión, sino en la economía del mundo antiguo ${ }^{1}$. Sin embargo, hay que considerar la gran cantidad de testimonios que se han perdido, como si los "archivos de la memoria", sólo nos dejaran atisbar por una ranura el inmenso espectáculo de la actividad social y económica que rodeaba al mundo de la miel y los productos melíferos. Queda siempre pendiente el esfuerzo de rehacer la verdadera historia, a través de los testimonios que daban cuenta de la vida cotidiana, como en este caso.

El estudio de los materiales ordinarios, que no fueron concebidos con la finalidad de que fueran observados por otros (las inscripciones públicas, p.e.), o para dar publicidad a una normativa (las leyes municipales, los senadoconsultos), son un rastro de vida latente, una prueba de la existencia o del interés de una actividad, sobre todo para los individuos que en ella participaban (restos de recipientes, de recibos, de contratos, de etiquetas, de instalaciones de trabajo). Dichos testimonios son decisivos para la reconstrucción de la economía y la vida social de una comunidad, son la base de la reconstrucción de la propia cultura material y del funcionamiento de una sociedad en su conjunto, de sus formas de subsistencia, de su alimentación y de su calidad de vida. Las abejas y la miel han tenido, en este orden, una relevancia singular en la historia de la Humanidad y queda mucho por hacer, para entender de forma acabada nuestro presente en este mismo contexto. Esta investigación, que adelanta ahora los primeros resultados, quiere señalar algunas de las direcciones en las que hay que seguir trabajando, para lograr una mejora de la regulación jurídica de las abejas, de la actividad agroeconómica dependiente de ellas, así como de los productos melíferos.

Se advierte, a día de hoy, una conciencia social cada vez más generalizada de la importancia de las abejas para el mantenimiento de los ciclos vitales y la biodiversidad. Una mirada retrospectiva al tratamiento jurídico, detallado y técnico, que la Jurisprudencia romana dedica a las abejas (D.41,1,5,2-3; Gai.2,68; Inst.2,1,14), es, una vía de aproximación imprescindible, que sirve, además, para valorar la importancia del tratamiento jurídico de todos los animales (insectos incluidos), considerados en su individualidad.

Precisamente la crisis sanitaria que padecemos como consecuencia del Covid-19, ha puesto de manifiesto que las catástrofes naturales, las enfermedades, las oscilaciones climáticas, los cambios en el gusto, son indicativos que transforman, a veces radicalmente, las condiciones materiales y espirituales -la vida en su complejidad-, de toda una sociedad ${ }^{3}$. En efecto, observamos hoy mejor que hace un año, antes de la pandemia, la relevancia de los productos de proximidad -de "Kilómetro 0"-, la importancia de la salud individual y colectiva y la necesidad de la preservación de nuestro entorno natural tal como lo conocemos.

En esta línea, hay dos cuestiones que conciernen directamente a las abejas y los productos melíferos: la cuestión de la propiedad y la regulación del comercio de la miel y productos derivados.

\footnotetext{
*Este trabajo es el punto de partida de un estudio más amplio y exhaustivo, que requiere de la colaboración de un equipo multidisciplinar. Expreso mi reconocimiento por la crítica constructiva y aportes de materiales, de Gerardo Caja, Jordi Serratosa, Miryam Olivera y Silvia Zanini, miembros del ICALP (UAB).

${ }^{1}$ Vid. por todos, BORTOLIN, R., Archeologia del miele (Mantova 2008) 188p.

${ }^{2}$ Siempre útil, en este sentido, la obra colectiva, coordinada por NICOLET, C. (Ed.), La mémoire perdue. À la recherche des archives perdues publiques et privées de la Rome Antique ( $1^{a}$ ed. Paris 1994; última ed. Paris 2019) DOI: 10400/books.psorbonne.25020

${ }^{3}$ HARPER, K., The Fate of Rome. Climate, Disease and the End of an Empire (Princeton 2017) 512p.
} 


\subsection{Propiedad}

La presencia de las abejas en muchos textos jurídicos romanos y otras fuentes históricas resulta de especial interés, pues el tratamiento que se les otorga conforma un régimen jurídico que ha permanecido prácticamente inalterado y reflejado en los principales Códigos occidentales, si bien la abundante normativa administrativa, superpuesta a la propiamente $\operatorname{civil}^{4}$, no ha podido eludir el punto de arranque de la cuestión acerca de la propiedad de los enjambres y las colmenas ${ }^{5}$, de la que depende el calificativo mismo de las abejas como animales silvestres o domesticados.

La cuestión central que tratan los textos jurídicos romanos en relación a las abejas, es la conservación de la propiedad del enjambre que migra -lo que se relaciona con la naturaleza jurídica del enjambre y, por ende, de las abejas-, y con el modo de adquisición de dicha propiedad, que se relaciona con otros dos principios centrales de la regulación jurídica de los animales, a saber: el estatuto jurídico de los animales, considerados hasta el presente como cosas en propiedad ${ }^{6} \mathrm{y}$, en estrecha relación con la propiedad, la debatida cuestión de la libertad natural de los animales ${ }^{7}$, que se relaciona con la afirmación en los textos de la naturaleza silvestre de las abejas (...apium quoque natura fera est, D.41,1,5,2 y 3$)^{8}$.

Por lo que se refiere a la propiedad, el punto debatido es la occupatio del enjambre que, en las épocas propicias para ello ${ }^{9}$, puede migrar a otra finca, lo que plantea el problema de si el dueño de la colmena conserva o no la propiedad de ese conjunto de abejas que se desplaza fuera de los límites de la finca que le pertenece ${ }^{10}$.

En este sentido, el Código civil español -al igual que el resto de los Códigos europeos y latinoamericanos se inspira en la configuración romana de la propiedad ${ }^{11}$, según la cual las cosas de mayor relevancia para el patrimonio de un individuo eran las llamadas cosas mancipables (res mancipi), entre las que se cuentan, los terrenos y casas en suelo de Italia, los esclavos y los animales de tiro y carga ${ }^{12}$, dichas cosas -que exigían para poder ser adquiridas de un acto formal -con o sin intervención del magistrado (mancipatio, in iure cessio, addictio)-, se distinguen de las cosas no mancipables (res nec mancipi), que son las de libre cambio, que pueden ser adquiridas sin necesidad de un acto formal que dote de eficacia al acto adquisitivo de la propiedad ${ }^{13}$, es decir, por medio de los llamados actos de "apropiación posesoria", entre los que se cuentan la simple entrega (traditio) -por eminencia ${ }^{14}$-, pero también la ocupación, la accesión o la especificación ${ }^{15}$. El Código civil no reproduce tal distinción, pero usa, sin embargo, la distinción parangonable a aquélla- entre cosas muebles e inmuebles, dado que el antiguo mancipium -fundamento de la distinción entre cosas mancipables y no mancipables- ${ }^{16}$, ya había perdido relevancia en el S.VI y no se incorpora a la Compilación justinianea ${ }^{17}$. Sí en cambio seguía teniendo sentido, en el momento de la Codificación, la distinción entre bienes inmuebles (o "bienes raíces") y muebles, como se advierte en los artículos $333^{18}$ y $335^{19}$ de nuestro actual Código civil.

${ }^{4}$ GARCIA GIMÉNEZ, A., La protección jurídica y administrativa de las abejas, en Actualidad Jurídica Ambiental 80 (2018) 2-33

${ }^{5}$ POLO TORIBIO, G., Abejas, enjambre, colmena: evolución histórico-jurídica a la luz del Fuero de Cuenca, Los derechos reales: actas del II Congreso Internacional y V Iberoamericano de Derecho Romano / coord. por Armando José Torrent Ruiz (2001) 211-231 ${ }^{6}$ GIMÉNEZ-CANDELA, M., Transición animal en España (Valencia 2019) 180ss.; esp.225-262

${ }^{7}$ GIMÉNEZ-CANDELA, M., Derecho Privado Romano (Valencia 2020, 2aed.) $\S \S 28,4$,a,i y 28,4,a,ii

${ }^{8}$ FILIP-FROSCHL, J., Apis natura fera est. Romanistische Anmerkungen zur besonderen Natur der Biene, en Scientia Iuris et Historiae, Festschrift für Peter Putzer zum 65. Geburtstag, Verlag Kovar, Eglinga.d.Paar, I (2004) 141-173

${ }^{9}$ GARCIA GIMÉNEZ, A., La protección jurídica y administrativa de las abejas, en Actualidad Jurídica Ambiental 80 (2018) 2-33

${ }^{10}$ MANTOVANI, D., I giuristi, il retore e le api. Ius controversum e natura nella Declamatio maior XIII, en Seminarios Complutenses de Derecho Romano y tradición romanística, 19 (2006) 205-283

${ }^{11}$ AA.VV., La Codificazione del Diritto dall'antico al moderno (Napoli 1998); KASER, M., KNÜTEL, R., Römisches Privatrecht (18. Auflage, München 2005).

${ }^{12}$ Gai.2,14a: “(...) et ea animalia quae collo dorsove domari solent, velut boves equi, muli, asini...” (=así como los animales que suelen domarse por el cuello o por el lomo, como los bueyes, los caballos, los mulos, los asnos).

${ }^{13}$ Gai.2,19: "Nam res nec mancipi ipsa traditione pleno iure alterius fiunt, si modo corporales sunt et ob id recipiunt traditionem" (=En efecto, las res nec mancipi se hacen de otro en plena propiedad por la simple entrega, siempre que sean corporales y por ello susceptibles de entrega).

${ }^{14}$ Por todos, WACKE, A., Das Besitzskonstitut als Übergabesurrogat in Rechtsgeschichte und Rechtsdogmatik. Ursprung, Entwicklung und Grenzen des Tradizionsprinzips im Mobiliarsachenrecht (Köln 1974)

${ }^{15}$ SEO, E., Der rechtsgeschäftliche Erwerb von Eigentum an beweglichen Sachen im Römischen Recht und in der römisch-rechtlichen Tradition (Münster 2004).

${ }^{16}$ Entre otros, BRETONE, M., I fondamenti del Diritto Romano: le cose e la natura (Roma-Bari 1998); DAUBERMANN, Die Sachgesamtheit als Gegenstand des klassischen römischen Rechts: vornehmlich unter dem Blickwinkel von Veränderungen in ihrer Zusammensetzung (Bern 1993)

${ }^{17}$ Siendo la literatura relativa extensísima, baste referir una exposición excelente de los avatares de la propiedad, hasta su inclusión en los Códigos actuales y, especialmente en el BGB alemán, con selecta bibliografía sobre el particular, que ofrece, WESENBERG, G., WESENER, G., Historia del Derecho Privado Moderno en Alemania y en Europa, trad. de la $4^{\mathrm{a}}$ ed. alemana (Wien-Köln-Graz 1985) por DE LOS MOZOS TOUYA, J.J. (Valladolid 1998) p.80ss., 198ss., 233ss., 296ss.

${ }^{18}$ Art. 333 Cc.: "Todas las cosas que son o pueden ser objeto de apropiación se consideran como bienes muebles o inmuebles".

${ }^{19}$ Art. 335 Cc.: "Se reputan bienes muebles los susceptibles de apropiación no comprendidos en el capítulo anterior, y en general, 
Así pues, los animales, configurados como cosas muebles, a partir de tal distinción básica, han pasado a nuestras legislaciones contemporáneas, diferenciados entre: los que sirven al hombre para trabajar la tierra y para el transporte y los que le sirven o proporcionan alimento (es decir, todos los demás, incluidas las abejas y las especies de caza) ${ }^{20}$.

Que el substrato ideológico de tal concepción es antropocéntrico y economicista ${ }^{21}$, no ha dejado de ponerse de relieve ${ }^{22}$.

Por lo demás, en la conformación jurídica de la propiedad, el punto de partida es la gran división de las cosas que hace la Jurisprudencia romana y, se encarga sobre todo de transmitir a las futuras generaciones un jurista de época adrianea denominado Gayo $^{23}$. En tal sentido, la clasificación gayana de las cosas que pueden ser objeto de una apropiación personal, deben distinguirse de aquellas que no pueden formar parte del patrimonio de un individuo ${ }^{24}$, por servir a los intereses de la comunidad, por estar dedicadas a los dioses, o por pertenecer al Pueblo Romano. Un concepto de propiedad en la que el valor de las cosas se mide por su utilidad, por la ventaja que al hombre le proporcionan, desde el punto de vista, es claro, de una sociedad eminentemente agraria ${ }^{25}$.

Conviene, sin embargo, hacer unas observaciones, que maticen la atribución, al Derecho Romano, de las consecuencias que se siguen de la calificación romana de los animales como cosas. Una primera puntualización, muy a tener en cuenta, es que la versión de los animales como cosas en propiedad de las que el hombre podría, hipotéticamente, hacer uso y abuso, no es propiamente una noción que pueda atribuirse al Derecho Romano; pues, en lo tocante a los animales, más bien, lo que queda claro, tras una aproximación a las fuentes clásicas, que aquí sería excesivamente prolijo detallar, es que el Derecho Romano, como fundamento del Derecho Civil Europeo, mantiene respecto a la naturaleza, a los seres vivos y, muy en especial respecto a los animales, una actitud de profundo respeto ${ }^{26}$.

Una segunda observación, que incide parcialmente en la ya formulada, es que el uso y abuso como prerrogativas de la propiedad que autorizaría al dueño "a disponer de la cosa del modo más absoluto", tal como aparece en el art. 544 del Código de Napoleón ${ }^{27}$, ha sido objeto de múltiples matizaciones, como bien se ha observado ${ }^{28}$, para pasar a ser un derecho debilitado e incluso fragmentado, como admite la mayor parte de la doctrina civilística.

Una tercera puntualización debe hacerse, a propósito de la afirmación de que la inclusión de los animales como cosas, dentro del sistema jurídico romano -y, por ello, en los sistemas jurídicos continentales, presenta al menos la ventaja de que los animales, de este modo pasan a tener un reconocimiento como realidad jurídica, como "un primer paso para entrar en la zona de protección del Derecho", lo que, como consecuencia, por ejemplo, habría protegido a los animales de los daños por terceros, a través de la lex Aquilia de damno. Otra ventaja sería que una realidad jurídica puede siempre sufrir variaciones, mejoras, cambios, supresiones. Esta argumentación, ha sido objeto de discusiones, sobre todo por quienes atribuyen todo lo malo que a los animales les ha ocurrido, como secuela de su inclusión como cosas en el ordenamiento jurídico romano.

La recepción de tales rasgos distintivos de la propiedad romana, y, como consecuencia, de la concepción de los animales como cosas, no sufrió - como se ha dicho- grandes variaciones en el proceso codificador. En efecto, los dos grandes parámetros de la configuración jurídica de los animales en nuestro Código civil, están contenidos en los artículos 465 y 610 . En el art. 465, se delinea lo que podría denominarse un régimen general

\footnotetext{
todos los que se pueden transportar de un punto a otro sin menoscabo de la cosa inmueble a la que estuvieren unidos".

${ }^{20}$ En el momento en que se codifica el Derecho Civil, no se piensa - evidentemente- en los animales de experimentación, los destinados a la peletería, los usados en espectáculos públicos para la diversión o los animales de granjas de producción.

${ }^{21}$ Cfr. LANATA, G., Antropocentrismo e cosmocentrismo nel pensiero antico, en CASTIGNONE, S., LANATA, G. (ed.), Filosofi e animali nel mondo antico (Pisa 1994) 35

${ }^{22}$ Por todos, CORNIL, G., Traité de la possession dans le droit romain: pour servir de base a une étude comparative des législations modernes (Paris 1905); CAPOGROSSI COLOGNESI, L., La terra in Roma antica: forme di proprietà e rapporti produttivi (Roma 1981); id., Proprietà e diritti reali: usi e tutela della proprietà fondiaria nel diritto romano (Roma 1999); BÖHR, R., Das Verbot der eigenmächtigen Besitzumwandlung im römischen Privatrecht: ein Beitrag zur rechtshistorischen Spruchregelforschung (Köln 2002).

${ }^{23}$ De las muchas ediciones de las Instituciones gayanas, por todos, vid. MANTHE, U., Gaius. Institutiones. Herausgegeben, Überstzung und Kommentar (Darmstadt 2004).

${ }^{24}$ Gai. 4,1: “(...) modo videamus de rebus; quae vel in nostro patrimonio sunt vel extra nostrum patrimonium habentur”; Gai.4,2:

${ }^{25}$ RATHBONE, D., Rural Economy and Society in Roman Italy (Cambridge University Press 2004); REMESAL RODRÍGUEZ, J., Heeresversorgung im frühen Prinzipat. Eine Art, die antike Wirtschaft zu verstehen. (Münster 2002); SCHEIDEL, W., von REDEN, S. (ed.) The Ancient Economy (Princeton 2002); FINLEY, M.I., Ancient Economy (California University Press 1999); DUNCANJONES, R., Structure \& Scale in the Roman Economy (Cambridge University Press 1990); WEBER, M., Wirtschaft und Gesellschaft: Grundri $\beta$ der verstehende Soziologie/Max Weber; besorgt von Johannes Winckelmann (Tübingen 1985)

${ }^{26}$ Vid. SÆLID GILHUS, I., Animals, Gods and Humans (London-New York 2006)

${ }^{27}$ Code civil, art. 544: "La propriété est le droit de jouir et de disposer des choses de la manière la plus absolue pourvu qu'on en fasse pas un usage prohibé par les lois ou par les règlements".

${ }^{28}$ RODOTA, S., El Derecho terrible (Madrid 1986)
}

12 Derecho Animal. Forum of Animal Law Studies, vol. 11/4 
$\mathrm{y}$, en el art. 610, se detalla la apropiación como forma de adquisición de las cosas (y, por ende, de los animales) que no tienen dueño (res nullius). Que el Code civil francés ignorara los avances científicos que, sobre la naturaleza de los animales, ya se habían producido en aquel tiempo, o la reflexión filosófica tendente a considerar a los animales como elementos integrantes de una naturaleza que merecía respeto, así como las corrientes de pensamiento que acentuaban la capacidad de sentir de los animales ${ }^{29}$, sino que en la Codificación se persistiera en la afirmación de los animales como cosas, equiparables a cualquier bien mueble, tuvo un influjo directo e innegable en el resto de las Codificaciones europeas y latinoamericanas ${ }^{30}$.

El artículo 465 del Cc. está redactado como a continuación se detalla:

"Los animales fieros sólo se poseen mientras se hallen en nuestro poder; los domesticados o amansados se asimilan a los mansos o domésticos, si conservan la costumbre de volver a la casa del poseedor".

La equiparación de los animales con las cosas se extrae tanto de la redacción del articulado, como de la ubicación sistemática del mismo ${ }^{31}$. La adopción del criterio romano del animus revertendi, aplicado a los animales domesticados o amansados, no deja de ser sorprendente en pleno siglo XIX. Por lo tocante al sentido primigenio de la expresión animus revertendi, conviene tener presente que los juristas romanos lo emplean como señuelo para verificar si sobre un animal se tiene o no la potestas, es decir el control fáctico y actual sobre él. Resulta curioso recordar que las gallinas, sobre las que no había unanimidad acerca de si tenían o no animus revertendi $i^{32}$, discurría entre los juristas romanos una animada polémica dirigida a establecer sí su naturaleza era salvaje o doméstica ${ }^{33}$; algo semejante puede decirse de la naturaleza de las abejas una vez migraba el enjambre.

Se emplea aquí el término posesión en el sentido de tenencia material (possessio naturalis) para distinguirla de la posesión civil (possessio civilis) o tenencia con ánimo de propietario. Sin entrar ahora en la trayectoria histórica de ambos conceptos, valga decir que en nuestro Código civil, se recoge dicha distinción romana, en los artículos 430 ss., haciendo extensivo al propietario, o al poseedor material de la cosa, las facultades inherentes al dominio como son el uso, el disfrute y la disposición, la cual incluye la facultad del dueño de extender su poder sobre lo suyo de manera omnímoda, sin más limitaciones que las que determine la ley ${ }^{34}$ : poder, pues, de enajenar, de gravar la cosa, de consumirla, de abandonarla, de destruirla o, en último término, de tratarla como el dueño lo considere oportuno sin que esté prevista, en principio, la justificación de un eventual maltrato.

Por lo que se refiere al artículo 610 del Cc. ${ }^{35}$, está concebido en los términos siguientes:

"Se adquieren por la ocupación los bienes apropiables por su naturaleza que carecen de dueño, como los animales que son objeto de la caza y de la pesca, el tesoro oculto y las cosas muebles abandonadas".

\footnotetext{
${ }^{29}$ BENTHAM, J., (London, 1748-1832), se considera el primer defensor del Bienestar Animal (“Animal Welfare”), en su obra, An Introduction to the Principles of Moral and Legislation, publicada en Londres en 1789 (año en que estalla la Revolución Francesa) y cuya última edición en inglés data de 2005 (Adamant Media Corporation), razonaba en los términos siguientes: que la capacidad de sufrir de los animales, más que su capacidad de hablar o razonar, era lo que les hacía merecedores de consideración y respeto: "What else is it that should trace the insuperable line? Is it the faculty of reason or perhaps the faculty of discourse? But a full-grown horse or dog is beyond comparison a more rational, as well as more conversable animal, than an infant of a day or a week or even a month old. But suppose they were otherwise, what would it avail? The question is not, Can they reason?, nor Can they talk? but, Can they suffer? Why should the law refuse its protection to any sensitive being? The time will come when humanity will extend its mantle over everything which breathes".

${ }^{30}$ Los Códigos civiles de Austria y Alemania, han excluido a los animales del concepto de cosa, como tendremos ocasión de mostrar; vid. infra, sub 2

${ }^{31}$ El artículo 465 Cc. cierra el capítulo III (de los efectos de la posesión), del título V que trata de la posesión, ubicado dentro del libro II: De los bienes, de la propiedad y de sus modificaciones.

${ }^{32} \mathrm{Cfr}$. Inst. 2,1,16; también, Inst.2,1,14 y 15, a propósito de la naturaleza-salvaje o no- de las abejas, pavos o palomas. En relación al animus revertendi, por todos, FILIP-FROSCHL, J., Cervi, qui in silvas ire et redire solent. Anmerkungen zu einem exemplum iuris, en Iurisprudentia universalis. Festschrift für Theo Mayer-Maly zum 70. Geburtstag, Hrsg. v. M. J. Schermaier (Köln, Weimar, Wien, Böhlau 2002) 191- 213

${ }^{33}$ Los tratados sobre los animales constituyeron desde Aristóteles el acercamiento científico a la naturaleza de los mismos, por excelencia. Muy mezclados con elementos de fantasía o imaginación, con datos no constatables, dichos tratados nutrieron la imaginación y la visión de los animales hasta bien entrado el S.XIX. Cfr. CAMASSA, G., Frammenti del bestiario pitagorico nella riflessione di Porfirio, en CASTIGNONE, S., LANATA, G (ed.), Filosofi e animali nel mondo antico (cit.) 92 ss.

${ }^{34}$ Art. 348 Cc.: "La propiedad es el derecho de gozar y disponer de una cosa, sin mas limitaciones que las establecidas en las leyes"; que reproduce a la letra el Code civil francés art. 544 : "La propriété est le droit de jouir et de disposer des choses de la manière la plus absolue pourvu qu'on en fasse pas un usage prohibé par les lois ou par les règlements".

${ }^{35}$ Nuestro texto, se inspira directamente en el art. 2279 del Code civil francés: "En fait de meubles, la possession vaut titre. Néanmoins, celui qui a perdu ou auquel il a été volé une chose peut la revendiquer pendant trois ans à compter du jour de la perte ou du vol, contre celui dans les mains duquel il la trouve ; sauf à celui-ci son recours contre celui duquel il la tient". Cfr. DROSS, W., Le singulier destin de l'article 2279 du Code civil, Revue trimestrielle de Droit civil, 1 (2006) 27ss.
} 
El artículo mencionado abre el título del Código civil, destinado a regular la adquisición de la propiedad mediante ocupación ${ }^{36}$, que se considera, a tenor de la redacción del art. 609, el modo ordinario de ser propietario $^{37}$. Es claro que tal redacción tiene que ver con la conocida distinción escolástica entre modos originarios y derivativos de adquirir la propiedad, que suele considerarse, a pesar de su inexactitud, como el vértice del que depende la compleja cuestión de la adquisición de la propiedad. En efecto, la ocupación (occupatio) es el acto por el que el adquirente, sin mediación de ningún otro agente, o la dependencia de otro acto antecedente, se apropia de una cosa haciéndola suya ${ }^{38}$. Lo que constituye un acto directo y primario de toma de posesión de algo. En tal sentido, siguiendo la tradición de la Jurisprudencia romana, que ejemplifica precisamente tales supuestos, nuestro Código considera apropriables por medio de la occupatio las cosas que no tienen dueño (res nullius), de forma permanente (los animales que se pueden cazar y pescar, así como las abejas cuando migran estacionalmente), o transitoria (el tesoro oculto y las cosas abandonadas) ${ }^{39}$.

La teoría de la ocupación y la relevancia que el Código le atribuye, tiene quizá hoy en día una justificación difícil, sin embargo, conviene no olvidar que, en el momento de la codificación del texto, era perfectamente coherente con el tipo de sociedad al que iba dirigida a regular, no muy diferente de la propia sociedad romana, que alumbró la construcción de esta categoría dogmática, a partir de la frecuencia casuística de su trayectoria. Por lo demás, es interesante recordar que los textos de la Jurisprudencia romana relativos a la ocupación, tuvieron una importancia decisiva para los grandes juristas del S.XVII, que vieron en ellos la forma de justificar la adquisición de las tierras de conquista, de la época de los grandes descubrimientos y expediciones al Nuevo Mundo: aquellos nuevos territorios eran también, para los descubridores y para los Reinos que financiaban tales viajes, res nullius, cosas carentes de dueño.

Que los animales, en nuestro caso las abejas, se inserten, en dicha teoría de la ocupación, como cosas asimiladas a los objetos de hallazgo, no es más que una extensión lógica del sistema jurídico que los acoge y del estatuto que se les concede. Coherentemente -y aunque pueden señalarse matices de tratamiento-, las crías de los animales se consideran frutos de la cosa productora y, por ello, se hacen de la propiedad del dueño de la cosa principal, por accesión ${ }^{40}$. Este es el sentido que en nuestro Código civil tiene el art. 355, párrafo 1, destinado a precisar qué se consideran frutos naturales:

"Son frutos naturales las producciones espontáneas de la tierra, y las crías y demás productos de los animales".

La simplificación propia (probablemente necesaria) del Código civil, sitúa en el mismo plano un huevo que un lechón, salvo para lo que se refiere al momento de su adquisición. Como es sabido, los frutos se adquieren por simple separación, lo que no rige para las crías de los animales que se consideran del dueño aún antes del parto, tal como dispone el artículo $357 \mathrm{Cc}$., en su segundo párrafo ${ }^{41}$.

La misma perspectiva se vislumbra en el artículo $499 \mathrm{Cc} .^{42}$ relativo al usufructo de rebaño o piara de ganado, en el que la exigencia de conservar "su forma y substancia", transcripción del principio salva rerum substantia que tanto juego casuístico dió a los juristas romanos, se traduce por la obligación de reemplazar con las crías las cabezas que mueran durante el año, por enfermedad o por efecto de los animales rapaces.

\subsection{Comercio de la miel y productos derivados}

Es bien sabido, por los muchos testimonios de las fuentes literarias (Verg. Georg.4, 256; Plin. NH 11,20;

\footnotetext{
${ }^{36}$ Cc. Libro III: "De los diferentes modos de adquirir la propiedad"; Título I: "De la ocupación".

37 Art. 609 Cc.: "La propiedad se adquiere por la ocupación” Así reza el primer párrafo, sin matices. A continuación discurre en su segundo párrafo el articulado: "La propiedad y los demás derechos sobre los bienes se adquieren y transmiten por la ley, por donación, por sucesión testada e intestada, y por consecuencia de ciertos contratos mediante la tradición".

${ }^{38}$ Cic. De offic. I, 7

${ }^{39}$ Cfr. por todos con lit. comparada, KNÜTEL, R., Der Wettlauf der Okkupanten, Usus Modernus pandectarum. Römisches Recht, Deutsches Recht und Naturrecht in der frühen Neuzeit. Klaus Luig zum 70. Geburstag (Köln,Weimar,Wien 2007) 75ss.

${ }^{40}$ Art. 353 Cc.: "La propiedad de los bienes da derecho por accesión a todo lo que ellos producen, o se les une o incorpora, natural o artificialmente". Art. 354 Cc.: "Pertenecen al propietario: $1^{\circ} \operatorname{los}$ frutos naturales. $2^{\circ}$ los frutos industriales. $3^{\circ} \operatorname{los}$ frutos civiles".

${ }^{41}$ Art. 357 Cc.: "No se reputan frutos naturales o industriales, sino los que están manifiestos o nacidos. Respecto a los animales, basta que estén en el vientre de su madre, aunque no hayan nacido".

${ }^{42}$ Art. 499 Cc.: "Si el usufructo se constituyere sobre un rebaño o piara de ganados, el usufructuario estará obligado a reemplazar con las crías las cabezas que mueran anual y ordinariamente, o falten por la rapacidad de animales dañinos. Si el ganado en que se constituyere el usufructo pereciese del todo, sin culpa del usufructuario, por efecto de un contagio u otro acontecimiento no común, el usufructuario cumplirá con entregar al dueño los despojos que se hubieren salvado de esta desgracia. Si el rebaño pereciere en parte, también por un accidente, y sin culpa del usufructuario, continuará el usufructo en la parte que se conserve. Si el usufructo fuere de ganado estéril, se considerará, en cuanto sus efectos, como si se hubiese constituido sobre cosa fungible".
} 
Colum, 9,7,5), epigráficas (CIL. I, 1409,4; IV, 1992; 5740 y 42; 9421; 10288) y papirológicas (PSI 428,8688; P. Lond. 2141, 19-23), que el comercio de la miel y los productos derivados de ella, es un aspecto capital para entender la economía del mundo antiguo. Sin embargo, la miel y productos melíferos no han solicitado la atención de los investigadores de la misma forma que otros alimentos o productos de comercio en la Antigüedad, como el vino o el aceite. La miel, a diferencia de estos, resulta más difícil de rastrear, pues los restos arqueológicos no son tan abundantes $\mathrm{y}$, dada su propia y frágil naturaleza, han desaparecido con mayor facilidad.

A pesar de las lagunas y ausencia de testimonios con los que nos enfrentamos, el estudio de esta cuestión es tan significativo como para merecer una revisión de las fuentes a disposición, desde una visión amplia y multidisciplinar de la que carecemos aunque tenemos apasionantes estudios de entomólogos y de apicultores ya desde el S. XVIII ${ }^{43}$. El interés no ha decrecido, al contrario, se ha acrecentado en las últimas décadas sobre todo entre los historiadores ${ }^{44}$.

La miel se transportaba en contenedores que, como el resto de productos de consumo, se etiquetaban para su identificación (tituli picti, graphiti) de los que se extraen interesantes conclusiones sobre las formas de alimentación y, especialmente del comercio, pues la miel, junto con el vino, el aceite y los cereales, se consideraba un alimento primario. Las noticias que se extraen de Plinio $(\mathrm{NH} 11,8,18)$ y sobre todo de Estrabón (Str.3,2,6), posicionan a la Bética como uno de los principales exportadores de miel ya en época augústea.

Actualmente, la miel representa en la UE una actividad de la que participan 600.000 apicultores que con 17 millones de colmenas producen unas 250.000 toneladas de miel al año, lo que es claramente insuficiente para atender la demanda de los consumidores de la UE, que precisa de la importación de otras 200.00 toneladas. Esta situación representa un $56 \%$ de autoabastecimiento, lo que si bien es inicialmente favorable para los apicultores, ha sufrido una clara degradación del mercado a causa de productos defectuosamente etiquetados, que confunden al consumidor.

La situación se ha abordado por parte de la UE en dos sucesivas Directivas ${ }^{45}$, en el sentido de tratar de fijar tanto la cuestión del origen botánico de la miel (floral, vegetal) y la denominación de origen, pues la multitud de regulaciones internas de los países está lejos de asegurar a los consumidores una información clara, transparente y completa.

\section{Las abejas en los Códigos continentales}

Que las abejas ostenten en su actual configuración jurídica, una naturaleza que se corresponde con la de los animales silvestres, aparece de forma taxativa en los principales Códigos continentales que siguen el modelo francés, derivado de la tradición romana ${ }^{46}$. La principal consecuencia de esta consideración de las abejas como pertenecientes a la fauna silvestre, es que sigue ofreciendo dificultades para la efectiva protección de éstas como individuos y, por lo demás tampoco entran en la órbita de la protección de los animales domésticos, que se caracteriza por ser una regulación, además de abundante, extremadamente detallada y prolija $^{47}$.

Pensar en una posible extensión a las abejas, de los niveles de protección que se da, por ejemplo, a las vacas lecheras, a las que (como al resto de animales de producción) el art. 13 TFUE considera "seres sentientes" (sentient beings), chocaría de momento con grandes impedimentos de carácter doctrinal. Aún es reciente la incorporación de los peces y cefalópodos a la órbita proteccionista de los seres sentientes y sigue siendo, para muchos, una afirmación chocante y de difícil aplicación que también la fauna acuática experimenta dolor o placer, por encima de sus reconocidas capacidades sensoriales ${ }^{48}$. Pues bien, pensemos por un momento que en breve la ciencia afirmara que también las abejas son seres sentientes y, por ello, como parámetro de tratamiento, deben serles extensivas las normas de bienestar que rigen para el resto de los animales llamados domésticos. Es claro que no se trata de clasificaciones, sino de aproximar a todos los seres vivos un nivel de tutela efectiva.

\footnotetext{
${ }^{43}$ RAY, P.A.P., Mémoire sur l'histoire des abeilles, en Journal de physique, 24 (1784) 117-129

${ }^{44}$ FERNÁNDEZ URIEL, P., Dones del cielo: abeja y miel en el Mediterráneo antiguo (Madrid 2011)

${ }^{45}$ Directiva 2014/63, del Parlamento Europeo y del Consejo de 15 de Mayo de 2014 (por la que se modifica la Directiva 2001/ 110/CE relativa a la miel (https://www.google.com/search?client=safari\&rls=en\&q=Directiva $+2014 / 63,+$ del + Parlamento + Europeo $+\mathrm{y}+\mathrm{del}+\mathrm{Consejo}+\mathrm{de}+15+\mathrm{d}$ e+Mayo+de+2014+(por+la+que+se+modifica+la+Directiva+2001/+110/CE+relativa+a+la+miel.\&ie=UTF-8\&oe=UTF-8

${ }^{46}$ FERNANDEZ DOMINGO, J.I., Las abejas y el Derecho (Madrid 2018) 192p.

${ }^{47}$ BROOM, D.M., Animal Welfare in the European Union (2017) https://www.europarl.europa.eu/supporting-analyses; PETERS, A., Animals in International Law (Leiden-Boston 2020) 236ss.

${ }^{48}$ GIMÉNEZ-CANDELA, M., SARAIVA, J.L., BAUER, H., The legal protection of farmed fish in Europe - analysing the range of EU legislation and the impact of international animal welfare standards for the fishes in European aquaculture, dA. Derecho Animal (Forum of Animal Law Studies) 11/1 (2020). DOI https://doi.org/10.5565/rev/da.460
} 
Esta verdadera laguna legal en el tratamiento de las abejas no ha llamado nunca la atención de los juristas, hasta que éstas no han comenzado a desaparecer de nuestro entorno de forma alarmante. Sólo por dicha razón, merecería ser reconsiderada una efectiva protección de las abejas -distinta a la existente-, además de una mejor gestión de la apicultura y de los productos melíferos, en los que reina la confusión más evidente. El tándem tutela-gestión de las abejas, como propósito de futuro para el legislador, debería ser inescindible.

En nuestro Código civil, este tratamiento aparece en el art. $612^{49}$, donde se atribuye al apicultor la propiedad del enjambre que migra tras una nueva reina y le otorga la facultad de conservar la propiedad del mismo, si inicia su persecución sin tardanza y de forma ininterrumpida. Por lo tanto, se trata de una reproducción exacta de la regulación romana, que causa extrañeza por el pintoresquismo de la misma y la carencia de una justificación práctica en la actualidad. Por lo demás, la actividad del apicultor se regula en el Real Decreto 209/2002, de 22 de febrero, por el que se establecen normas de ordenación de las explotaciones apícolas $^{50}$.

En idéntica situación respecto a las abejas, se encuentran otros Códigos civiles de nuestro entorno, por no hacer referencia aquí a los Códigos civiles de Latinoamérica. Me refiero, entre otros, al Codice Civile italiano $^{51}$, al Code Rural de Francia ${ }^{52}$, al Código civil (ZGB) de Suiza ${ }^{53}$ y al Código civil (BGB) alemán ${ }^{54}$

\section{Las abejas y la biodiversidad}

La biodiversidad de los insectos está amenazada en todo el mundo. En 2019 se publicó un examen exhaustivo acerca de la disminución de los insectos de todo el mundo, así como acerca de la evaluación sistemática de los factores subyacentes a este fenómeno alarmante ${ }^{55}$. El mencionado estudio, del que se extraen algunas de las cifras y consideraciones aquí reflejadas, revela tasas importantes de disminución de las poblaciones de insectos y se afirma en el mismo, que tales índices pueden llevar a la extinción del $40 \%$ de las especies de insectos del mundo en las próximas décadas.

En los ecosistemas terrestres, los lepidópteros, himenópteros (abejas, avispas, abejorros) y coleópteros (escarabajos peloteros) parecen ser los taxones más afectados. Los grupos de insectos afectados no sólo incluyen especies que ocupan nichos ecológicos particulares, sino también muchas especies comunes y generalistas. Al mismo tiempo, la abundancia de un pequeño número de especies, extraño a esta clasificación tradicional, está aumentando; se trata de especies adaptables y comunes que están ocupando los nichos vacíos dejados por los que están disminuyendo.

Los principales impulsores de la disminución de las referidas especies parecen ser los siguientes factores, relacionados a continuación en orden de importancia:

a) la pérdida de hábitats y la implantación de la agricultura intensiva y la urbanización;

b) la contaminación, principalmente la causada por los plaguicidas y fertilizantes sintéticos;

c) los factores biológicos, incluidos los patógenos y las especies introducidas; $\mathrm{y}$, por último

d) el cambio climático. Este último factor es particularmente importante en las regiones tropicales, pero sólo afecta a una minoría de especies en climas más fríos y en entornos montañosos de zonas templadas.

Que la preocupación en el ámbito científico sobre la situación de las abejas es un hecho, se evidencia en el creciente número de publicaciones que, precisamente en 2020, han aparecido ${ }^{56}$. El nexo común de la mayoría de ellas es poner de relieve la carencia de acciones concretas, tanto estatales como globales ${ }^{57}$, que

\footnotetext{
${ }^{49} \mathrm{Cc}$. art 612: "El propietario de un enjambre de abejas tendrá derecho a perseguirlo sobre el fundo ajeno, indemnizando al poseedor de éste el daño causado. Si estuviere cercado, necesitará el consentimiento del dueño para penetrar en él." // "Cuando el propietario no haya perseguido, o cese de perseguir el enjambre dos días consecutivos, podrá el poseedor de la finca ocuparlo o retenerlo."

${ }^{50}$ BOE 62, de 13 de marzo de 2002 https://www.boe.es/eli/es/rd/2002/02/22/209

${ }^{51} \mathrm{Cc}$.it. art. 924: "Il proprietario di sciami di api ha diritto di inseguirli sul fondo altrui' ma debe indennità per il danno cagionato al fondo; se non li ha inseguiti entro due giorni o ha cessato durante due giorni di inseguirli, può prenderli e ritenerli il proprietario del fondo."

${ }^{52}$ Code Rural. Art. L. 211-9: "Le propriétaire d'un essaim a le droit de le réclamer et de s'en ressaisir, tant qu'il n'a pas cessé de le suivre; autrement l'essaim appartient au propriétaire du terrain sur lequel il s'est fixé."

${ }^{53}$ ZGB 719.3: "Bienenschwärme werden dadurch, dass sie auf fremden Boden gelangen, nicht herrenlos."

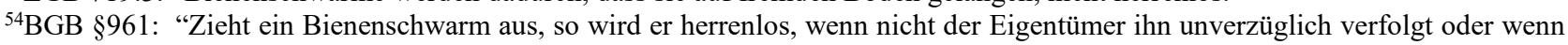
der Eigentümer die Verfolgung aufgibt."

55 SANCHEZ-BAYO, F., WYCKHUIS, K., A.G., Worlwide decline of the entomofauna. A Review of its drivers, en Biological Conservation, 232 (2019) 8-27. DOI: https://doi.org/10.1016/j.biocon.2019.01.020

${ }^{56}$ Entre enero de 2019 y diciembre de 2020, se han publicado más de 100 artículos científicos relativos a las abejas, como puede constatarse en la base de datos de Science Direct, www.sciencedirect.com

${ }^{57}$ ORR, M.C., HUGUES, A.C., CHESTERS, D., PICKERING, J., DONGZU, C., ASCHE, J.S., Global Patterns and Drivers of Bee 
pongan freno a la creciente desaparición de las abejas; no saber hacer frente con los medios usuales a la convivencia entre especies; así como los efectos del Covid-19 y otras enfermedades infecciosas, conforme al principio de "One Health", que tiene como objetivo estudiar cuestiones epidemiológicas fundamentales ${ }^{58}$. Dado que, tanto las abejas silvestres como las administradas, son polinizadores esenciales de los cultivos y las flores silvestres, la salud de las mismas tiene un impacto directo en la salud humana y ambiental y, a través de ellas, pueden abordarse cuestiones referidas a la inmunología ecológica desde una mejor aproximación a la incidencia en la salud de los polinizadores.

El Parlamento Europeo ha definido expresamente el sector de la apicultura como "esencial" para la UE, ya que realiza una importante contribución a la sociedad, tanto económica (unos 14.200 millones de euros al año) como medioambiental, manteniendo el equilibrio ecológico y la biodiversidad, ya que el $84 \%$ de las especies vegetales y el $76 \%$ de la producción de alimentos en Europa dependen de la polinización entomófila (Parlamento Europeo, 2019) ${ }^{59}$.

Por otra parte, en los últimos años ha aumentado la conciencia pública sobre la disminución de las poblaciones de insectos polinizadores, de forma que en el año 2019 los ciudadanos europeos pusieron en marcha una iniciativa ${ }^{60}$ para proteger a las abejas, pidiendo a la Comisión que eliminara el uso de pesticidas en la agricultura de la UE para conservar y restablecer las poblaciones de insectos y que aumentara el apoyo a los agricultores en la transición hacia prácticas agronómicas más sostenibles.

A nivel estatal, España no sólo es uno de los países europeos con mayor número de especies de abejas, sino también el segundo productor de miel de abeja doméstica (Apis mellifera) en Europa, después de Rumania. Por lo tanto, es evidente que la apicultura y la agricultura relacionada con los cultivos dependientes de la polinización son sectores clave para la economía española, por lo que España corre el riesgo de ser uno de los países que puede sufrir más intensamente las consecuencias del alarmante descenso de los insectos polinizadores ${ }^{61}$.

En suma, se necesita urgentemente un replanteamiento de las prácticas agrícolas actuales, en particular una importante reducción del uso de plaguicidas y su sustitución por prácticas más sostenibles y de base ecológica, para frenar o invertir las tendencias actuales, permitir la recuperación de las poblaciones de insectos en disminución y salvaguardar los servicios vitales que prestan a los ecosistemas.

\section{Referencias}

- AA.VV., La Codificazione del Diritto dall'antico al moderno (Napoli 1998)

- BENTHAM, J., An Introduction to the Principles of Moral and Legislation (London 1789)

- BORTOLIN, R., Archeologia del miele (Mantova 2008)

- BÖHR, R., Das Verbot der eigenmächtigen Besitzumwandlung im römischen Privatrecht: ein Beitrag zur rechtshistorischen Spruchregelforschung (Köln 2002)

- BRETONE, M., I fondamenti del Diritto Romano: le cose e la natura (Roma-Bari 1998)

- BROOM, D.M., Animal Welfare in the European Union https://www.europarl.europa.eu/supporting-analyses

- CAMASSA, G., Frammenti del bestiario pitagorico nella riflessione di Porfirio, en CASTIGNONE, S., LANATA, G. (ed.), Filosofi e animali nel mondo antico (Pisa 1994) 92 ss.

- CAPOGROSSI COLOGNESI, L., La terra in Roma antica: forme di proprietà e rapporti produttivi (Roma 1981)

- CAPOGROSSI COLOGNESI, L., Proprietà e diritti reali: usi e tutela della proprietà fondiaria nel diritto romano (Roma 1999)

- CORNIL, G., Traité de la possession dans le droit romain: pour servir de base a une étude comparative des législations modernes (Paris 1905)

\footnotetext{
Distribution, en Current Biology 31 (2020) 1-8. DOI: https://doi.org/10.1016/j.cub.2020.10.053

${ }^{58}$ WILFERT, L., BROWN, M.J.F., DOUBLET, V., One Health implications of infectious diseases of wild and managed bees, en Journal of Invertebrate Pathology (2020) DOI: https://doi.org/10.1016/j.jip.2020.107506 ; PUMAROLA I BATLLE, M., Enfermedad Animal, zoonosis y "One Health": lo que hemos aprendido los veterinarios a lo largo de la historia, en dA.Derecho Animal 11/4 (2020). DOI: https://doi.org/10.5565/rev/da.520

59 Noticias del Parlamento Europeo: ¿Qué hay detrás de la disminución de las abejas y otros polinizadores?. https://www.europarl.europa.eu/news/es/headlines/society/20191129STO67758/que-hay-detras-de-la-disminucion-de-las-abejas-yotros-polinizadores

${ }^{60}$ Iniciativa Ciudadana Europea $"$ iSalvemos a las abejas y a los agricultores! Hacia una agricultura respetuosa con las abejas para un medio ambiente sano" (30 de septiembre de 2019)

${ }^{61} \mathrm{La}$ aparición de la avispa asiática (Vespa velutina), una especie alóctona invasora que pone en grave peligro la supervivencia de las abejas y que avanza de forma imparable, extendiéndose por gran parte del país, especialmente en Galicia, la Cornisa Cantábrica y Cataluña, también es un dato que conviene tomar en consideración.
} 
- DAUBERMANN, Die Sachgesamtheit als Gegenstand des klassischen römischen Rechts: vornehmlich unter dem Blickwinkel von Veränderungen in ihrer Zusammensetzung (Bern 1993)

- DROSS, W., Le singulier destin de l'article 2279 du Code civil, en Revue trimestrielle de Droit civil, 1 (2006) 27ss.

- DUNCAN-JONES, R., Structure \& Scale in the Roman Economy (Cambridge University Press 1990)

- FERNANDEZ DOMINGO, J.I., Las abejas y el Derecho (Madrid 2018)

- FERNÁNDEZ URIEL, P., Dones del cielo: abeja y miel en el Mediterráneo antiguo (Madrid 2011)

- FILIP-FROSCHL, J., Apis natura fera est. Romanistische Anmerkungen zur besonderen Natur der Biene, en, Scientia Iuris et Historiae, Festschrift für Peter Putzer zum 65. Geburtstag, Verlag Kovar, Eglinga.d.Paar, I (2004) 141-173

- FILIP-FROSCHL, J., Cervi, qui in silvas ire et redire solent. Anmerkungen zu einem exemplum iuris, en Iurisprudentia universalis. Festschrift für Theo Mayer-Maly zum 70. Geburtstag, Hrsg. v. M. J. Schermaier (Köln, Weimar, Wien, Böhlau 2002) 191- 21

- FINLEY, M.I., Ancient Economy (California University Press 1999)

- GARCIA GIMÉNEZ, A., La protección jurídica y administrativa de las abejas, en Actualidad Jurídica Ambiental, 80 (2018) 2-33

- GIMÉNEZ-CANDELA, M., Transición animal en España (Valencia 2019)

- GIMÉNEZ-CANDELA, M., SARAIVA, J.L., BAUER, H., The legal protection of farmed fish in Europe - analysing the range of EU legislation and the impact of international animal welfare standards for the fishes in European aquaculture, dA. Derecho Animal (Forum of Animal Law Studies) 11/1 (2020). DOI https://doi.org/10.5565/rev/da.460

- HARPER, K., The Fate of Rome. Climate, Disease and the End of an Empire (Princeton 2017)

- KASER, M., KNÜTEL, R., Römisches Privatrecht (18. Auflage, München 2005)

- KNÜTEL, R., Der Wettlauf der Okkupanten, Usus Modernus pandectarum. Römisches Recht, Deutsches Recht und Naturrecht in der frühen Neuzeit. Klaus Luig zum 70. Geburstag (Köln,Weimar,Wien 2007) 75ss.

- LANATA, G., Antropocentrismo e cosmocentrismo nel pensiero antico, en CASTIGNONE, S., LANATA, G. (ed.), Filosofi e animali nel mondo antico (Pisa 1994) 35

- MANTHE, U., Gaius. Institutiones. Herausgegeben, Überstzung und Kommentar (Darmstadt 2004)

- MANTOVANI, D., I giuristi, il retore e le api. Ius controversum e natura nella Declamatio maior XIII, en Seminarios Complutenses de Derecho Romano y tradición romanística, 19 (2006) 205-283

- NICOLET, C. (ed.), La mémoire perdue. À la recherche des archives perdues publiques et privées de la Rome Antique (1 ${ }^{a}$ ed. Paris 1994; última ed. Paris 2019) DOI: 10400/books.psorbonne.25020

- ORR, M.C., HUGUeS, A.C., CHESTERS, D., PICKERING, J., DONGZU, C., ASCHE, J.S., Global Patterns and Drivers of Bee Distribution, en Current Biology 31 (2020) 1-8. DOI: https://doi.org/10.1016/j.cub.2020.10.053

- PETERS, A., Animals in International Law (Leiden-Boston 2020)

- POLO TORIBIO, G., Abejas, enjambre, colmena: evolución histórico-jurídica a la luz del Fuero de Cuenca, Los derechos reales: actas del II Congreso Internacional y V Iberoamericano de Derecho Romano / coord. por Armando José Torrent Ruiz (2001) 211-231

- PUMAROlA i BATLle, M., Enfermedad Animal, zoonosis y "One Health": lo que hemos aprendido los veterinarios a lo largo de la historia, en dA.Derecho Animal 11/4 (2020). DOI: https://doi.org/10.5565/rev/da.520

- RATHBONE, D., Rural Economy and Society in Roman Italy (Cambridge University Press 2004)

- RAY, P.A.P., Mémoire sur l'histoire des abeilles, en Journal de physique, 24 (1784) 117-129

- REMESAL RODRÍGUEZ, J., Heeresversorgung im frühen Prinzipat. Eine Art, die antike Wirtschaft zu verstehen. (Münster 2002); Scheidel \& von Reden (ed.) The Ancient Economy (Princeton 2002)

- RODOTA, S., El Derecho terrible (Madrid 1986)

- SÆLID GILHUS, I., Animals, Gods and Humans (London-New York 2006)

- SANCHEZ-BAYO, F., WYCKHUIS, K. A.G., Worlwide decline of the entomofauna. A Review of its drivers, en Biological Conservation 232 (2019) 8-27. DOI: https://doi.org/10.1016/j.biocon.2019.01.020

- SCHEIDEL, W., von REDEN, S. (ed.) The Ancient Economy (Princeton 2002) 
- SEO, E., Der rechtsgeschäftliche Erwerb von Eigentum an beweglichen Sachen im Römischen Recht und in der römisch-rechtlichen Tradition (Münster 2004)

- WACKE, A., Das Besitzskonstitut als Übergabesurrogat in Rechtsgeschichte und Rechtsdogmatik. Ursprung, Entwicklung und Grenzen des Tradizionsprinzips im Mobiliarsachenrecht (Köln 1974)

- WEBER, M., Wirtschaft und Gesellschaft: Grundriß der verstehende Soziologie/Max Weber; besorgt von Johannes Winckelmann (Tübingen 1985)

- WeSEnBerG, G., WESENER, G., Historia del Derecho Privado Moderno en Alemania y en Europa, trad. de la $4^{a}$ ed. alemana (Wien-Köln-Graz 1985) por DE LOS MOZOS TOUYA, J.J. (Valladolid 1998) p.80ss., 198ss., 233ss., 296ss.

- WILFERT, L., BROWN, M.J.F., DOUBLET, V., OneHealth implications of infectious diseases of wild and managed bees, en Journal of Invertebrate Pathology(2020) DOI: https://doi.org/10.1016/j.jip.2020.107506 